\title{
Protecting Personal Privacy under the Environment of Big Data
}

\author{
Dandan Dong and Xuejun Zhou* \\ College of Mathematics and Physics, Huanggang Normal University, Huanggang 438000, Hubei, China \\ ${ }^{*}$ Corresponding author
}

\begin{abstract}
Personal privacy of citizens under the environment of big data is a hot spot of social concern. This paper focuses on the issue of the commercialization and sharing of personal data. A simple analytic hierarchy process is used to rationally divide the risk of privacy and build an evaluation system. The privacy equivalent model is then established through the construction of membership functions in fuzzy theory and service level of Vickrey-Clark-Groves (VCG). At last, based on the problem of liability commitment, corresponding recommendations are studied under the data leakage mechanism.
\end{abstract}

Keywords—risk degree; vickrey-clark-groves (VCG); membership function; privacy pricing model

\section{INTRODUCTION}

With the reform of the times, internet technology has developed rapidly and brought convenience to many people. The use and dependence of electronic communication and social media is a common phenomenon, but while people enjoy it, they also suffer from the leakage of personal data. There are differences in the privacy of personal data industry. Personal privacy data mining and classification are widely spread in different industries, and it brings the infinite value for the human and the nation. However, some criminals use personal privacy data to threaten users' information security. The number of personal data is too numerous to protect the individual data, and excessive protection will inhibit the development of the Times. How to deal with privacy data reasonably and make full use of privacy data is the most important task in today's world on the premise of low risk to users.

\section{THE Establishment AND SOLUTION OF MODEL}

\section{A. Determine the Weight of Each Influencing Factor}

1) Determine risk parameters and establish hierarchical model

In risk assessment, this paper classifies risks to determine the parameters which affect the risk model. The privacy risk is divided into three aspects, i.e., social media, financial transaction, and health care. Meanwhile, three aspects can be divided into six specific as: WeChat, Weibo, social networking sites, direct financial transaction, indirect financial transaction and health care. A hierarchy model [1] diagram of privacy risk is built by the analytic hierarchy process, as shown in Fig. 1 .

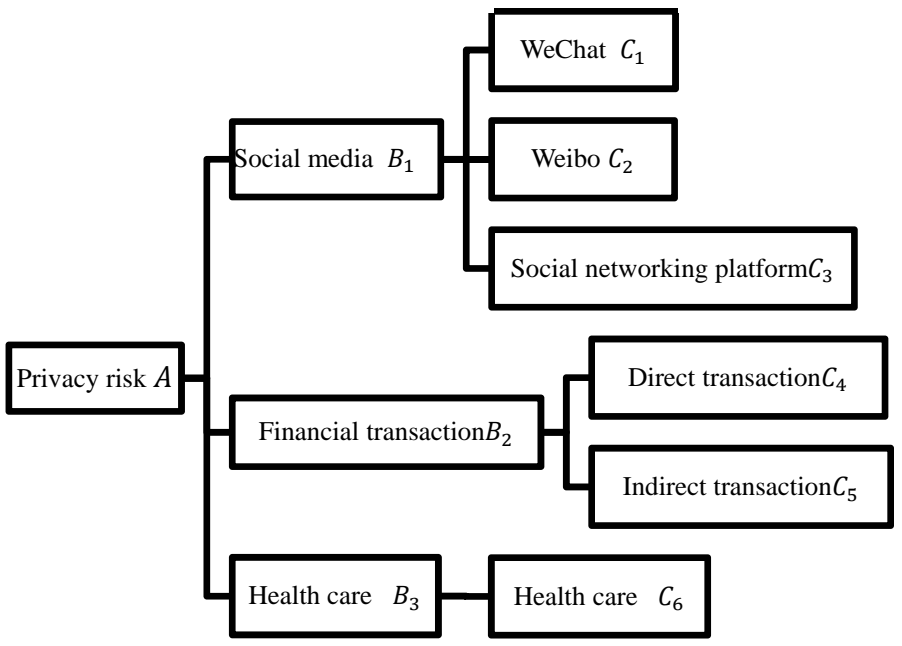

FIGURE I. PRIVACY RISK HIERARCH MODEL

2) Establish the judgment matrix for comparison between every two indexes

The judgment matrix of privacy risk is constructed based on hierarchy model. The method of the judgment matrix for comparison between every two indexes and 9 scale scaling is carried out from the basic layer $\mathrm{A}$. The degree of risk is expressed in Arabia 1-9, and the comparison results of element $\mathrm{i}$ and element $\mathrm{j}$ are expressed by $B_{i j}$. The basic description is given as Table 1.

TABLE I. THE MEANING OF SCALE 1-9

\begin{tabular}{|c|c|}
\hline The comparison of element $\boldsymbol{i}$ and element $\boldsymbol{j}$ & Scale \\
\hline The element $B_{\mathrm{i}}$ is as important as the element $B_{\mathrm{j}}$ & Note $B_{\mathrm{i} j}=1$ \\
\hline The element $B_{\mathrm{i}}$ is tiny- important as the element $B_{\mathrm{j}}$ & Note $B_{\mathrm{i} \mathrm{j}}=3$ \\
\hline The element $B_{\mathrm{i}}$ is low- important as the element $B_{\mathrm{j}}$ & Note $B_{\mathrm{i} \mathrm{j}}=5$ \\
\hline The element $B_{\mathrm{i}}$ is middle-important as the element $B_{\mathrm{j}}$ & Note $B_{\mathrm{i} \mathrm{j}}=7$ \\
\hline The element $B_{\mathrm{i}}$ is high- important as the element $B_{\mathrm{j}}$ & Note $B_{\mathrm{i} \mathrm{j}}=9$ \\
\hline The element $B_{\mathrm{i}}$ is close to the element $B_{\mathrm{j}}$ & $B_{\mathrm{i} \mathrm{j}}=2,4,6,8$ \\
\hline
\end{tabular}




\section{3) Computing weight vector}

The comparison matrix between the target layer $A$ and the standard layer $B$ can be written as follow

$$
\left(\begin{array}{lll}
B_{11} & B_{12} & B_{13} \\
B_{21} & B_{22} & B_{23} \\
B_{31} & B_{32} & B_{33}
\end{array}\right)
$$

The each row product of the comparison matrix can be computed and noted $M_{\text {i }}$

$$
M_{i}=\sum_{j=1}^{n} B_{i j}, i=1,2, \cdots, n
$$

The $n$ square of $M_{\mathrm{i}}$ is as follow

$$
\bar{W}_{i}=\sqrt[n]{M_{i}}, i=1,2, \cdots, n
$$

The vector $\bar{W}$ can be obtained

$$
\bar{W}=\left[\bar{W}_{1}, \bar{W}_{2}, \cdots, \bar{W}_{n}\right]
$$

Normalization of vector $W_{i}$ is as follow

$$
W_{i}=\frac{\bar{W}_{i}}{\sum_{i=1}^{n} \bar{W}_{i}}
$$

It can be seen that $W_{1}, W_{2}, \ldots W_{\mathrm{n}}$ are the weight vector of $B_{1}, B_{2}, \ldots B_{\mathrm{n}}$.

\section{4) Martix one-time validation}

The random one-time index RI values are shown in Table 2.

TABLE II. THE VALUE OF THE RANDOM ONE-TIME INDEX RI

\begin{tabular}{|c|c|c|c|c|c|c|c|c|c|}
\hline Order & 1 & 2 & 3 & 4 & 5 & 6 & 7 & 8 & 9 \\
\hline RI & 0 & 0 & 0.58 & 0.90 & 1.12 & 1.24 & 1.32 & 1.41 & 1.49 \\
\hline
\end{tabular}

The maximum eigenvalue of the comparison matrix can be expressed as

$$
\lambda_{\max }=\sum_{i=1}^{n} \frac{(A W)_{i}}{n W_{i}}
$$

The random one-time index RI value can be written as

$$
C I=\frac{\lambda_{\max }-n}{n-1},\left(C R=\frac{C I}{R I}\right)
$$

The calculated results are compared with the random onetime index values in Table 2. The smaller the index value, the more effective the weight vector is, so is the greater the risk.

\section{B. Model Complement and Improvement}

In this problem, the hierarchy process needs to satisfy the consistency of the comparison matrix. If the consistency is not satisfied, this model will fail. Fuzzy mathematics ${ }^{[2]}$ as a new branch of mathematics can increase the reliability of risk evaluation results when evaluating many factors.

1) The rules of evaluation

A set of risk levels

$$
V=\left\{V_{1}, V_{2}, V_{3}, V_{4}\right\}
$$

where $V_{1}$ denotes tiny-risk, $V_{2}$ low-risk, $V_{3}$ middle-risk and $V_{4}$ high-risk.

2) Establish the membership function of ach index

$$
r\left(v_{k}\right)\left(c_{i}\right)=\left\{\begin{array}{cc}
0 & v_{0}<c_{i}<v_{i k} \\
\frac{v_{i k}-c_{i}}{v_{i k}-v_{i(k-1)}} & v_{i k}<c_{i}<v_{i(k+1)} \\
1 & v_{i(k+1)}<c_{i}<v_{i(k+2)}
\end{array}\right.
$$

The standard matrix can be expressed as

$$
r_{i}=\left[r\left(v_{1}\right)\left(c_{i}\right), r\left(v_{2}\right)\left(c_{i}\right), r\left(v_{3}\right)\left(c_{i}\right), r\left(v_{4}\right)\left(c_{i}\right),\right]
$$

Based on weighted average fuzzy comprehensive evaluation, a model of supply chain risk can be expressed as

$$
B=\left(\partial_{1}, \partial_{2}, \partial_{3}, \partial_{4}\right) \mathrm{O}\left(B_{1}, B_{2}, B_{3}, B_{4}\right)=\left(b_{1}, b_{2}, b_{3}, b_{4}\right)
$$

\section{3) The solution of the membership function}

Assigning 4 levels of the risk, we order 4 risk degrees from high to low is $1,2,3$, and 4 . Then the formula for calculating the comprehensive of comprehensive risk index can be obtained

$$
y=1 \times b_{1}+2 \times b_{2}+3 \times b_{3}+4 \times b_{4}
$$

That $\gamma \in[0,1]$ means that the risk is tiny-risk, $\gamma \in[1,2]$ low-risk, $\gamma \in[2,3]$ middle-risk, and $\gamma \in[3,4]$ high-risk. 


\section{4) "Paired comparison method" for privacy pricing model}

Privacy pricing is affected in many ways. In model 1 , it is divided into WeChat, Weibo, social network, direct financial transaction, indirect financial transaction and health care. And on this base, they interact with each other and build a privacy cost model ${ }^{[3]}$

$$
\Gamma=f\left(X_{1}, X_{2}, X_{3}, X_{4}, X_{5}, X_{6}\right)
$$

where $X_{\mathrm{i}}$ represents the influencing factor.

Ordering

$$
U=\left\{X_{1}, X_{2}, X_{3}, X_{4}, X_{5}, X_{6}\right\}
$$

where $X_{i}$ represents the privacy pricing elements. The evaluation vector can be expressed as follow

$$
V=\left\{V_{1}, V_{2}, V_{3}, V_{4}\right\}
$$

The comprehensive evaluation of privacy pricing can be expressed as follow

$$
\Gamma=A \circ R
$$

The ascending (semi-trapezoidal) distribution of membership function in fuzzy theory ${ }^{[4]}$ is selected, and the specific membership function ${ }^{[5]}$ is determined by the following formula:

$$
\begin{gathered}
\mu_{i}(x)=\left\{\begin{array}{cc}
1 & x \leq x_{i 1}\left(x \geq x_{i 2}\right) \\
\frac{x-x_{i 1}}{x_{i 1}-x_{i 2}} & x_{i 1}<x{ }_{i 2}\left(x_{i 1}>x>x_{i 2}\right) \\
0 & x \geq x_{i 2}\left(x \leq x_{i 2}\right)
\end{array}\right. \\
\mu_{j}(x)=\left\{\begin{array}{cc}
\mid \frac{x-x_{i(j-1)} \mid,}{x_{i 1}-x_{i 2} \mid} & x_{(i-1)(j-1)} \leq x \leq x_{i j}\left(x_{j(x-1)} \geq x_{i j}\right) \\
\mid \frac{x_{i(j+1)}}{x_{i 1}-x_{i 2} \mid} & x_{i j}<x<x_{i(j+i)}\left(x_{(i-1) j}>x_{i j}\right)
\end{array}\right. \\
\mu_{n}(x)=\left\{\begin{array}{cc}
0, & x \leq x_{i(j-1)}, x \geq x_{i(j+1)}\left(x \geq x_{i(j+1)}, x<x_{i(j+1)}\right) \\
\mid \frac{x-x_{i(n-1)}}{x_{i n}-x_{i(n-1)} \mid} & x_{i(n-1)}<x_{i n}, x_{i(n-1)}>x>x_{i n} \\
0 & x \leq x_{i(n-1)}, x \geq x_{i(n-1)}
\end{array}\right.
\end{gathered}
$$

According to the regional eigenvalues and expert advices, the importance of any two of the six factors is compared, and the evaluation matrix ${ }^{[6]}$ is obtained as follow:

$$
C_{66}=\left[\begin{array}{llllll}
C_{11} & C_{12} & C_{13} & C_{14} & C_{15} & C_{16} \\
C_{21} & C_{22} & C_{23} & C_{24} & C_{25} & C_{26} \\
C_{31} & C_{32} & C_{33} & C_{34} & C_{35} & C_{36} \\
C_{41} & C_{42} & C_{43} & C_{44} & C_{45} & C_{46} \\
C_{51} & C_{52} & C_{53} & C_{54} & C_{55} & C_{56} \\
C_{61} & C_{62} & C_{63} & C_{64} & C_{65} & C_{66}
\end{array}\right],
$$

where $C_{i j}$ represents the ratio of the factor $i$ to the importance of the factor $j$, this paper takes the median value to the expert. Then, the weight of each factor is obtained by using the two comparison matrix algorithm in the AHP.

\section{5) Expert survey method to obtain data}

The qualitative approach to risk assessment is to use a literal or descriptive level to illustrate the extent of the risk. In this paper, we use the expert survey method ${ }^{[7]}$ to obtain the data and analyze the important discriminant (see Table 3).

TABLE III. SUMMARY OF INPORTANCE COMPARISON

\begin{tabular}{|c|c|c|c|c|c|c|}
\hline & $\begin{array}{c}\text { WeCh } \\
\text { at }\end{array}$ & $\begin{array}{c}\text { Wei } \\
\text { bo }\end{array}$ & $\begin{array}{c}\text { Social } \\
\text { networki } \\
\text { ng } \\
\text { platform }\end{array}$ & $\begin{array}{c}\text { Dirct } \\
\text { transacti } \\
\text { on }\end{array}$ & $\begin{array}{c}\text { Indirec } \\
\mathbf{t} \\
\text { transa } \\
\text { ction }\end{array}$ & $\begin{array}{c}\text { Healt } \\
\mathbf{h} \\
\text { care }\end{array}$ \\
\hline Wechat & 1 & 3 & 3 & 5 & 9 & 7 \\
\hline Webo & $1 / 3$ & 1 & 2 & 5 & 7 & 5 \\
\hline $\begin{array}{c}\text { Social } \\
\text { networking } \\
\text { platform }\end{array}$ & $1 / 3$ & $1 / 2$ & 1 & 3 & 5 & 3 \\
\hline $\begin{array}{c}\text { Direct } \\
\text { transaction }\end{array}$ & $1 / 5$ & $1 / 5$ & $1 / 3$ & 1 & 5 & 3 \\
\hline $\begin{array}{c}\text { Indirect } \\
\text { transaction }\end{array}$ & $1 / 9$ & $1 / 7$ & $1 / 5$ & $1 / 5$ & 1 & $1 / 3$ \\
\hline $\begin{array}{c}\text { Health } \\
\text { care }\end{array}$ & $1 / 7$ & $1 / 5$ & $1 / 3$ & $1 / 3$ & 3 & 1 \\
\hline
\end{tabular}

Substituting it into the discriminant matrix, we can obtain

$$
A=\left[\begin{array}{cccccc}
1 & 3 & 3 & 5 & 9 & 7 \\
\frac{1}{3} & 1 & 2 & 5 & 7 & 5 \\
\frac{1}{3} & \frac{1}{2} & 1 & 3 & 5 & 3 \\
\frac{1}{5} & \frac{1}{5} & \frac{1}{3} & 1 & 5 & 3 \\
\frac{1}{9} & \frac{1}{7} & \frac{1}{5} & \frac{1}{5} & 1 & \frac{1}{3} \\
\frac{1}{7} & \frac{1}{5} & \frac{1}{3} & \frac{1}{3} & 3 & 1
\end{array}\right]
$$




\section{6) Solution of the model}

Supposed $n$ factors, which are recorded as $L_{1}, L_{2}, L_{3}, \ldots, L_{\mathrm{n}}$, and their weights for those factors are $W_{1}, W_{2}, W_{3}, \ldots, W_{n}$. Assuming that there are $n$ bidders, and through $a_{1}, a_{2}, a_{3}, \ldots, a_{n}$, we can get that the greater the weight of the risk factor. First, the optimal match was obtained, and each bidder was given a factor with different risk weight. As this time, $a_{1}$ was matched with $L_{1}$, and $a_{n}$ was matched with $L_{n}$ (supposed that these factors are ranked in order of weight). Next, we can set the example of $L_{k}$ and calculate the VCG price $a_{k+1}$ paid by the bidder for $L_{k}$ with the risk weight $W_{k}$. We remove $W_{k}$ and $a_{k}$, then calculate the total value of the remaining matches

$$
\sum=a_{1} w_{1}+\ldots+a_{(k-1)} W_{(K-1)}+\ldots+a_{n} W_{n}(k>1, k=2,3,4 . .)
$$

The bidder who has bid for $a_{k+1}$ will be reevaluated to match the sum of the value matching

$$
\sum_{2}=a_{1} W_{2}+a_{(k-1)} W_{k}+\ldots+a_{n} W_{n}
$$

The VCG price $X_{k}$ that the bidder $L_{k}$ pays for the $a_{k}$ can be expressed as follow

$$
X_{k}=\sum_{2}-\sum_{1}
$$

In this problem, we use the VCG price of service level to determine the specific price of different weights for different weights. First, we calculate the eigenvectors of all kinds of influence factors, and then obtain the maximum eigenvalue $\lambda_{\max }=6.3630$ of the comparison matrix and the one-time index $\mathrm{CI}=0.0726$. Therefore, the smaller of the index value, the more effective of the weight vector is. We could ask the one-time index compared with random one-time index, and can find that the value is small, so the greater of the risk of the corresponding risk level is very high, which illustrates the feasibility of selecting the index data corresponds to the conclusion can be verified.

We studied the weight of the WeChat, Weibo, social networking, direct and indirect transaction and health care in Table 4.

TABLE IV. RISK INDICES FOR ALL ASPECTS

\begin{tabular}{|c|c|c|c|c|c|c|}
\hline & WeChat & Weibo & $\begin{array}{c}\text { Social } \\
\text { networking } \\
\text { platform }\end{array}$ & $\begin{array}{c}\text { Dirct } \\
\text { transaction }\end{array}$ & $\begin{array}{c}\text { Indirect } \\
\text { transaction }\end{array}$ & $\begin{array}{c}\text { Health } \\
\text { care }\end{array}$ \\
\hline Weight & 0.2315 & 0.1929 & 0.1900 & 0.1763 & 0.1442 & 0.0651 \\
\hline
\end{tabular}

Therefore, the privacy pricing model can be written as

$$
\Gamma=f\left(0.2436 X_{1}+0.203 X_{2}+0.1856 X_{3}+0.1518 X_{4}+0.0682 X_{5}+0.2 X_{6}+\xi\right)(25)
$$

\section{The PROBlem of Responsibility UNDER THE DATA LEAKAGE MECHANISM}

The privacy protection of personal data application is a significant social problem under the environment of big data. It also involves many fields such as morality, law, industry technology and so on. The abuse of data, the low level of information security technology, and low security factors can lead to the frequent loss of privacy, and the risk of data leakage has become an important factor that can't be ignored in privacy cost pricing. Because the original model does not consider the influence of external factors such as privacy leakage, this cascading event will cause data disclosure, which will have a great impact on the original risk cost pricing model. Therefore, it will result in the original risk pricing model not being accurate enough. Privacy leaks will cause the information owner's data to circulate without the authorization of privacy owners, which not only increases the risk of the data disclosure itself, but also causes the loss of privacy owners. Therefore, we should be compensated according to the value of the loss data. The traceability mechanism can quickly find the source of privacy leakage, and punish severely the party responsible for the disclosure. Forcing the data controllers to enhance their sense of responsibility of personal data pricing protection from the system, which is conducive to prevent the occurrence of privacy leakage from the source. In view of the risk of privacy leakage, we identify the responsible party through four aspects, such as traceability technical standard system, information registration system, traceability supervision system, and traceability information reward and punishment system. Based on the stakeholder perspective, this paper explores the issue of the privacy information's responsibility to be stolen by the network.

\section{CONCLUSION}

This paper makes a preliminary study of personal privacy issues under the environment of big data. The personal privacy sources are divided into six aspects: WeChat, social networking platform, direct financial transactions and health care. Using the analytic hierarchy, we assess the risk in six aspects and build a corresponding evaluation system. Next, on the basis of the evaluation system, we introduce membership functions in fuzzy theory. Through membership functions with VCG service mechanisms, we build a privacy pricing model. Personal privacy flows into the market as a commodity, and on the premise of meeting market supply and demand, it can generate huge impact on countries and individuals. Finally, aiming at the problem of liability bearing under the data leakage mechanism, this paper gives corresponding suggestions to make personal privacy data towards sharing, public welfare and commercialization.

\section{ACKNOWLEDGMENT}

This study was funded by Hubei Province Undergraduate Innovative Training Project (Name: Protecting personal privacy under the environment of big data) 


\section{REFERENCES}

[1] F. He. "Application of analytic hierarchy process in construction bidding”. Southwest Jiao Tong University, 2014. (in Chinese).

[2] J. Wu , Z. Cai, C. Hu, and J. Cao. "State evaluation of relay protection based on membership function of fuzzy normal distribution". Power system protection and control, 2012,40 (05): 48-52. (in Chinese).

[3] R. Zhu. "Study on the quality evaluation of the double ear hearing sound of a cooling fan.”. Harbin Institute of Technology, 2017. (in Chinese)

[4] H. Huang and J. Chen. "Research on the improvement mechanism of purchasing portfolio auction research". System engineering theory and practice, 2007 (11): 43-47. (in Chinese)

[5] Z. Wang, R. Ma, Y. Ma, and Y. Li. "Using the membership function method for the analysis of drought resistance of Lespedeza". Arid land resources and environment, 2013,27 (09): 119-123. (in Chinese)

[6] C. Yu. "Analysis of enterprise multi product pricing strategy with network externalities". University of electronic technology, 2009. (in Chinese)

[7] J. Liang and Z. Hou. "A comprehensive method for the combination of AHP method and neural network". System engineering theory and practice, 2001 (03): 59-63. (in Chinese). 